\title{
Design of a Proportional Observer Based on the ARX-Laguerre Model
}

\author{
Hassene BEDOUI*, Tarek GARNA, Kamel BEN OTHMAN, Hassani MESSAOUD \\ LARATSI, National Engineering School of Monastir, \\ University of Monastir, Monastir, TUNISIA \\ hassenebedoui@gmail.com; \{tarek.garna;kamel.benothman;hassani.messaoud\}@enim.rnu.tn \\ * Corresponding author
}

\begin{abstract}
ARX-Laguerre representation is recently built to model the dynamics of complex processes [1, 2]. The ARX-Laguerre models have proven their ability to accurately suit the behavior of systems. In this work, the model is exploited to diagnose the system by detecting its defaults. In this paper we build a proportional observer based on the ARX-Laguerre model. Therefore, the designed observer exploits the inputs and outputs of the Laguerre-ARX model to reconstruct the Laguerre filter outputs. The observer gain is calculated to ensure a fast asymptotic convergence of the estimation error. A simulation example is achieved to illustrate the ability of the proposed approach to estimate the Laguerre filter outputs.
\end{abstract}

Keywords: Diagnosis, ARX-Laguerre, proportional observer, SISO system, LMI, FDI.

\section{Introduction}

The dynamics of physical processes are often modeled by mathematical relations.These relations are generally differential equations or state representations dedicated exclusively to theoretical models, transfer functions or regressive representations (ARX, ARMAX, Arimax). Recently, Bouzraraet al [1,2] proposed a new representationable to model the dynamics of complex physical processes. The proposed representation called ARX-Laguerre achieves a significant complexity reduction compared to the linear ARX standard model. The principle of the ARX-Laguerre model is based on filtering the input and the output of theARX standard model by the orthogonal Laguerre functions. This new representation is useful in case of observers based diagnosis for state representation modeled systems. Generally, observers used for linear systems are often Luenberger-ones or with proportional gain [3]. In this work, we propose a proportional observer which can reconstructthe Laguerre filter outputs from the inputs and outputs of the ARX-Laguerre model.The reconstruction is achieved througha comparison between the outputs of the estimated Laguerre filters and the real outputs. The ARX-Laguerre based diagnosis is anew approach since the proposed model is recent $[1,2]$.

This work is presented as follows: A theoretical study on the new ARX-Laguerre linear modeling is presented in Section 1, followed in the second section by developing its recursive representation used in the observer design. In the third section, we present the synthesis of proportional observer exploiting the ARXLaguerre model. This step is characterized by the development of the observer structure, the synthesis of gain matrices and Lyapunov ones. In addition, the conditions of existence of the observer are established. A work on improving the performance of the new observer is achieved in the same section. In the last section, the synthesis technique of the proportional observer is applied to an illustrative example showing the effectiveness of the developed method.

\section{ARX-Laguerre Model}

A strictly causal discrete time system can be described by an ARX model as:

$$
y(k)=\sum_{j=1}^{\infty} h_{a}(j) y(k-j)+\sum_{j=1}^{\infty} h_{b}(j) u(k-j)
$$

where $u(k)$ and $y(k)$ are the system input and output respectively, $n a$ and $n b(n b \leq n a)$ are the model order associated to the output and the input respectively, $h_{a}$ and $h_{b}$ are the model parameters. In this paragraph, we detail the development of the ARX model on independent Laguerre orthonormal bases to provide a reduced parameter number model. Due to stability condition in the meaning of Bounded Input Bounded Output (BIBO) criterion, the coefficients $h_{a}$ and $h_{b}$ satisfy:

$$
\sum_{j=1}^{\infty}\left|h_{a}(j)\right|<\infty, \text { and } \sum_{j=1}^{\infty}\left|h_{b}(j)\right|<\infty
$$


and the ARX model (1) can be written as:

$$
y(k)=\sum_{j=1}^{\infty} h_{a}(j) y(k-j)+\sum_{j=1}^{\infty} h_{b}(j) u(k-j)
$$

Such that:

$$
\left\{\begin{array}{l}
h_{a}(j)=0, \text { if } j>n a \\
h_{b}(j)=0, \text { if } j>n b
\end{array}\right.
$$

As the coefficients $h_{a}$ and $h_{b}$ are summable, they belong to Lebesgue space $\ell^{2}[0, \infty)$ and since the Laguerre orthonormal functions form an orthonormal basis in such space [4], the coefficients $h_{a}$ and $h_{b}$ can be decomposed on two independent Laguerre bases $\mathfrak{I}_{a}$ and $\mathfrak{I}_{b}$ with respect to the output and the input respectively as:

$$
\begin{aligned}
& h_{a}(j)=\sum_{n=0}^{\infty} g_{n, a} \ell_{n}^{a}(j) \\
& h_{b}(j)=\sum_{n=0}^{\infty} g_{n, b} \ell_{n}^{b}(j)
\end{aligned}
$$

Where and $\ell_{n}^{b}$ are the orthonormal functions of Laguerre bases $\mathfrak{I}_{a}$ and $\beta=0.5$ respectively $g_{n, a}$ and $g_{n, b}$ are the Fourier coefficients. By substituting the linear combinations of relation (5) in the ARX model defined by (3) and (4), the resulting model, entitled the ARX-Laguerre is written as:

$$
y(k)=\sum_{n=0}^{\infty} g_{n, a} x_{n, y}(k)+\sum_{n=0}^{\infty} g_{n, b} x_{n, u}(k)
$$

where $x_{n, y}$ and $x_{n, u}$ are the filtered output and the filtered input respectively by Laguerre functions such as:

$$
x_{n, y}(k)=\sum_{n=0}^{\infty} \ell_{n}^{a}(j) y(k-j)=\ell_{n}^{a}(k) * y(k)
$$

$$
x_{n, u}(k)=\sum_{n=0}^{\infty} \ell_{n}^{b}(j) u(k-j)=\ell_{n}^{b}(k) * u(k)
$$

where $*$ is the convolution product. In practice, the infinite series in (6) can be truncated to a finite orders $N a$ and $N b$ as to obtain the truncated ARX-Laguerre model:

$$
y(k)=\sum_{n=0}^{N a-1} g_{n, a} x_{n, y}(k)+\sum_{n=0}^{N b-1} g_{n, b} x_{n, u}(k)
$$

The parameter number of the new black-box linear ARX-Laguerre model is:

$$
M=N a+N b
$$

Then, comparing the relations (1) and (9), a significant reduction of the parameter number is satisfied if $N a+N b<n a+n b$. This parametric complexity reduction with respect to the classical ARX model is proved theoretically in [2]. Furthermore, it was shown in [1,2] that the Laguerre ARX-model is characterized by the following recursive representation:

$\left\{\begin{array}{l}X(k+1)=A X(k)+b_{y} y(k)+b_{u} u(k) \\ y(k)=c^{T} X(k)\end{array}\right.$

such that:

$X \in \mathbb{R}^{M}$ is a discrete vector contains the filters outputs.

$A \in \mathbb{R}^{M \times M}$ the $M$-dimentional matrix defined as :

$A=\left(\begin{array}{cc}A_{y} & 0_{N a, N b} \\ 0_{N b, N a} & A_{u}\end{array}\right)$

where $0_{N b, N a}$ and $0_{N a, N b}$ are the null matrices of dimensions $(\mathrm{Nb} \times \mathrm{Na})$ and $(\mathrm{Na} \times \mathrm{Nb})$ respectively and $A_{y}$ and $A_{u}$ are two square matrices of dimension $N a$ and $N b$ respectively:

$$
\begin{aligned}
& A_{y}=\left[\begin{array}{ccccc}
\xi_{a} & 0 & 0 & \cdots & 0 \\
1-\xi_{a}^{2} & \xi_{a} & 0 & \cdots & 0 \\
-\xi_{a}\left(1-\xi_{a}^{2}\right) & 1-\xi_{a}^{2} & \xi_{a}^{2} & \cdots & 0 \\
\vdots & \vdots & \vdots & \ddots & \vdots \\
\left(-\xi_{a}\right)^{N a-2}\left(1-\xi_{a}^{2}\right) & \left(-\xi_{a}\right)^{N a-3}\left(1-\xi_{a}^{2}\right) & \left(-\xi_{a}\right)^{N a-4}\left(1-\xi_{a}^{2}\right) & \cdots & \xi_{a}
\end{array}\right] \\
& \left.A_{u}=\mid \begin{array}{ccccc}
\xi_{b} & 0 & 0 & \cdots & 0 \\
1-\xi_{b}^{2} & \xi_{b} & 0 & \cdots & 0 \\
-\xi_{b}\left(1-\xi_{b}^{2}\right) & 1-\xi_{b}^{2} & \xi_{b}^{2} & \cdots & 0 \\
\vdots & \vdots & \vdots & \ddots & \vdots \\
\left(-\xi_{b}\right)^{N b-2}\left(1-\xi_{b}^{2}\right) & \left(-\xi_{b}\right)^{N b-3}\left(1-\xi_{b}^{2}\right) & \left(-\xi_{b}\right)^{N b-4}\left(1-\xi_{b}^{2}\right) & \cdots & \xi_{b}
\end{array}\right]
\end{aligned}
$$


$b_{y} \in \mathbb{R}^{M}$ and $b_{u} \in \mathbb{R}^{M}$ the $M$-dimensional vectors defined as:

$$
\begin{aligned}
& b_{y}=\sqrt{1-\xi_{a}^{2}}\left|\begin{array}{c}
1 \\
-\xi_{a} \\
\left(-\xi_{a}\right)^{2} \\
\vdots \\
\left(-\xi_{a}\right)^{N a-1} \\
0_{N b, 1}
\end{array}\right| \\
& b_{u}=\sqrt{1-\xi_{b}^{2}}\left|\begin{array}{c}
0_{N a, 1} \\
1 \\
-\xi_{b} \\
\left(-\xi_{b}\right)^{2} \\
\vdots \\
\left(-\xi_{b}\right)^{N b-1}
\end{array}\right|
\end{aligned}
$$

where for $i=a, b, 0_{N i, 1}$ is the null vector of dimension $\mathrm{Ni}$.

$c^{T} \in \mathbb{R}^{M}$ is the parameters vector containing all the Fourier coefficients.

The representation (11) can be rewritten under the following form:

$$
\left\{\begin{aligned}
X(k+1) & =\left(A+b_{y} c^{T}\right) X(k)+b_{u} u(k) \\
y(k) & =c^{T} X(k)
\end{aligned}\right.
$$

\section{Synthesis of an ARX-Laguerre Observer}

\subsection{Designing a proportional observer}

The construction of an observer for theARXLaguerre model is possible by providing a correcting of the estimation error between the real output $y(k)$ and the model output $\hat{y}(k)$.

A proportional observer may be defined as follows:

$$
\left\{\begin{aligned}
\hat{X}(k+1) & =\left(A+b_{y} c^{T}\right) \hat{X}(k)+b_{u} u(k)+ \\
& +L(y(k)-\hat{y}(k)) \\
\hat{y}(k) & =c^{T} \hat{X}(k)
\end{aligned}\right.
$$

Equation (18) can be written as follows:

$$
\left\{\begin{aligned}
\hat{X}(k+1) & =\left(A+b_{y} c^{T}-L c^{T}\right) \hat{X}(k)+ \\
& +b_{u} u(k)+L y(k) \\
\hat{y}(k)= & c^{T} \hat{X}(k)
\end{aligned}\right.
$$

where $L$ is a column vector of dimension $M$, representing the gain of the proportional observer to calculate.
To ensure a good estimate of the vector of the filter outputs $\hat{X}(k)$ and the vector of the system output $\hat{y}(k)$, it must be ensured when designing the observer that the difference between the filter outputs and their estimations tend to zero as $k$ tends to infinity. This is true if the eigenvalues of the matrix $\left(A+b_{y} c^{T}-L c^{T}\right)$ are in the unit circle of the complex plane [5]. The observer gain $L$ can be determined by the pole placement technique if the following theorem is satisfied [6]:

Theorem 1: The eigenvalues of $\left(A+b_{y} c^{T}-L c^{T}\right)$ can be arbitrarily set if and only if the pair $\left(\left(A+b_{y} c^{T}\right), c^{T}\right)$ is observable.

If the pair $\left(\left(A+b_{y} c^{T}\right), c^{T}\right)$ is observable then the user has a wide margin of freedom to set the observer gain $L$.

The observer gain is generally chosen so that the eigenvalues of the matrix $\left(A+b_{y} c^{T}-L c^{T}\right)$ being in the unit circle of the complex plane, and that the real part of the eigenvalues are larger, in absolute value, than the real part of the eigenvalues of the matrix $\left(A+b_{y} c^{T}\right)$.

The estimation error between the output filters of the ARX-Laguerre model and the observer is defined as follows:

$$
e(k)=X(k)-\hat{X}(k)
$$

The dynamics of the estimation error:

$$
\begin{aligned}
& e(k+1)=X(k+1)-\hat{X}(k+1) \\
& e(k+1)=\left(\left(A+b_{y} c^{T}\right) X(k)+b_{u} u(k)\right)-\cdots \\
& \left(\left(A+b_{y} c^{T}-L c^{T}\right) \hat{X}(k)+b_{u} u(k)+L c^{T} X(k)\right)
\end{aligned}
$$

then

$$
e(k+1)=\left(A+b_{y} c^{T}-L c^{T}\right) e(k)
$$

To ensure convergence of the estimation error to zero, we have to choose the matrix $L$.

The convergence conditions of the estimation error are based on the following theorem of Tanaka et al. [7], [8].

Theorem 2 [9]: the observer (18) is asymptotically stable if there is a Lyapunov matrix $P$ symmetric and positive definite satisfying the following inequality:

$$
\left(A+b_{y} c^{T}-L c^{T}\right)^{T} P+P\left(A+b_{y} c^{T}-L c^{T}\right)<0
$$




\subsection{Improved performance of the observer}

The stability conditions given by the theorems 1 and 2 guarantee the convergence of the estimation error to zero, i.e. the convergence of the observer but don't introduce any effect on the rate of convergence of the observer [10], [11], [12].

Conventional tools for solving LMI may solve the inequalities (28) and (29), which leads to find the two unknowns $\mathrm{P}$ and $G$. The deduction of the value of $L$ is then evident using the formula:

$$
L=P^{-1} G
$$

\section{Illustrative Example}

By performing the decomposition rules of ARXLaguerre, we propose the following model:

$$
\left\{\begin{aligned}
X(k+1) & =A X(k)+b_{y} y(k) b_{u} u(k) \\
y(k) & =c^{T} X(k)
\end{aligned}\right.
$$

The numerical values of the matrices of the state equations are as follows for $M=4$ :

$$
\begin{aligned}
& A=\left[\begin{array}{llll}
0.4 & 0 & 0 & 0 \\
0.84 & 0.4 & 0 & 0 \\
0 & 0 & 0.7 & 0 \\
0 & 0 & 0.51 & 0.7
\end{array}\right] ; \quad b_{y}=\left[\begin{array}{c}
0.9135 \\
-0.3666 \\
0 \\
0
\end{array}\right] ; \\
& b_{u}=\left[\begin{array}{c}
0 \\
0 \\
0.7141 \\
-0.4999
\end{array}\right] ; c=\left[\begin{array}{c}
-1.3677 \\
-0.6682 \\
0.4727 \\
1.8136
\end{array}\right] .
\end{aligned}
$$

To ensure rapid convergence of the observer, we use the pole placement technique. The poles of the observer must be within a selected region of the complex plane LMI.

The chosen LMI region is the intersection of a disk with center $(0,0)$ of radius $\beta$ and the left half plane bounded by a straight abscissa equal to $-\alpha(\alpha>0)$. The observer has their eigenvalues in this region, if there exists a matrix $P$, symmetric, and defined as positive in (25) and (26).

Equation (25) and (26) are nonlinear in the variables $L$ and $P$. To solve them through solving tools LMI, we consider the following variable $G$ defined as follows:

$G=P L$

Using equation (27) the inequalities (25) and (26) can be written as (28) and (29).

The system input $u(k)$ is represented by Figure 1.

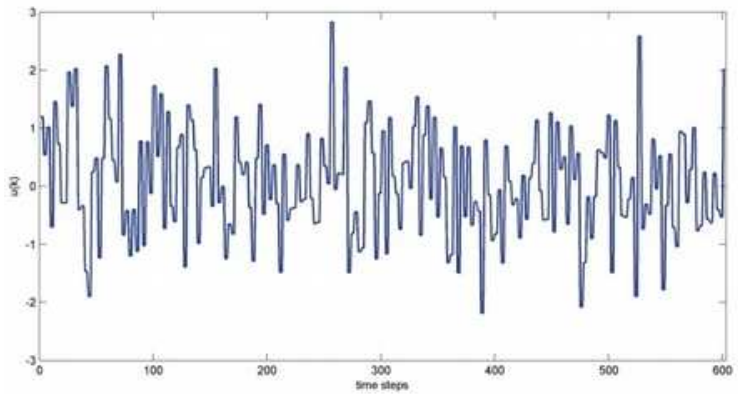

Figure 1. Input signal $u(k)$

Let assume that the system is affected by a default between the iterations 201 and 252.The output of the faulty system is then between these two instants. Figure 2 shows the system output with the default signal.

By proposing the proportional observer (18) and according to the convergence conditions of the estimation error and the stability of the observer, we seek to determine the gain of the observer $L$. The stability of the estimation error and the synthesis of the gain $L$ are provided by solving linear matrix inequalities (LMI).

By choosing $\beta=0.5$, the observer gain is:

$$
\begin{aligned}
& \left(\mid \begin{array}{cc}
-\beta P & \left(A+b_{y} c^{T}-L c^{T}\right)^{T} P \\
P\left(A+b_{y} c^{T}-L c^{T}\right) & -\beta P
\end{array}\right)<0 \\
& \left(A+b_{y} c^{T}-L c^{T}\right)^{T} P+P\left(A+b_{y} c^{T}-L c^{T}\right)+2 \alpha P<0 \\
& \left(\left(\begin{array}{cc}
-\beta P & A^{T} P+\left(b_{y} c^{T}\right)^{T}-\left(c^{T}\right)^{T} G \\
P A+P b_{y} c^{T}-G c^{T} & -\beta P
\end{array}\right)<0\right. \\
& A^{T} P+P A+\left(b_{y} c^{T}\right)^{T} P+P b_{y} c^{T}-\left(c^{T}\right)^{T} G-G c^{T}+2 \alpha P<0
\end{aligned}
$$




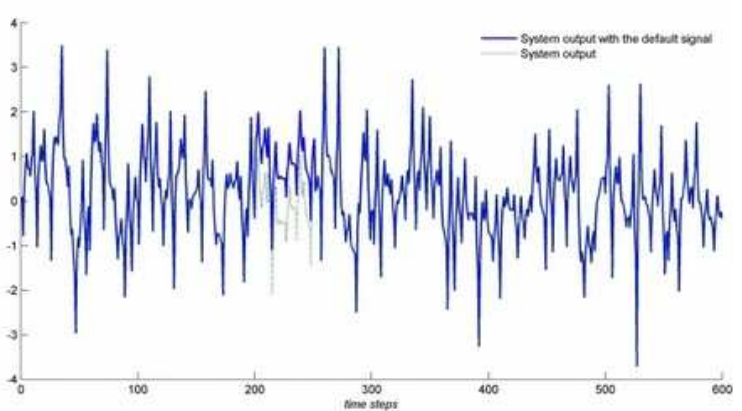

Figure 2. System output with the default signal

$$
L=\left[\begin{array}{c}
-1.1872 \\
-18.6333 \\
6.0701 \\
-8.5020
\end{array}\right] .
$$

After the gain synthesis, the observer is able to deliver an estimate of the output $y(k)$ and compare it later with ARX-Laguerre model output to detect any default in the model.

Figure 3 shows the output of theARX-Laguerre model and its estimated using the observer.

A residual signal is generated to detect a default that affect the system. Figure. 4 shows the evolution of the residual signal $r(k)$. It indicates a default when it significantly away from zero. Furthermore, we illustrate in Figure 5 the evolution of the estimated outputs vector of Laguerre filters $\hat{X}(k)$ and the real vector $X(k)$.

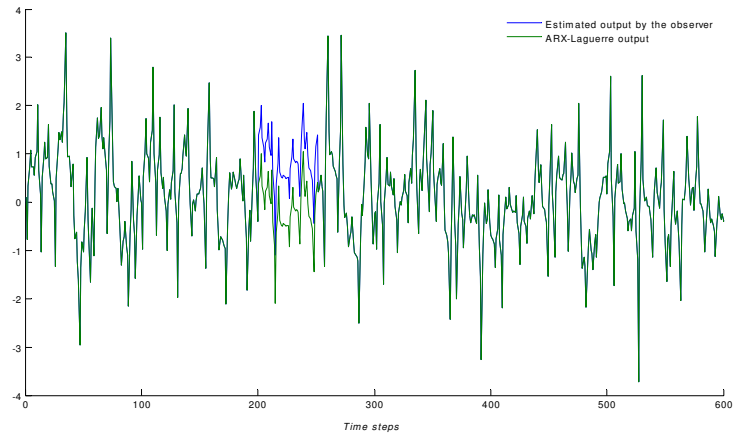

Figure 3. Output of the ARX-Laguerre model and its estimated by the observer

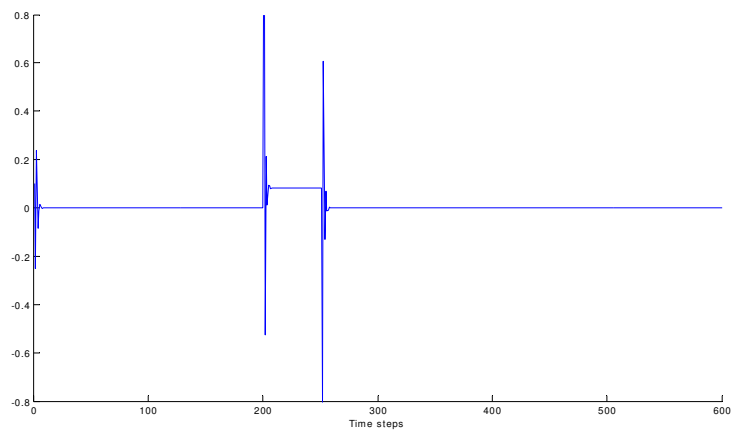

Figure 4. Evolution of the residual signal $r(k)$

Initially, the residual signal indicates some oscillations however there is a default.In fact the oscillations are due to the initial conditions of the observer. Note that the generated residual signal indicates a default between iterations 201 and 252. One the default disappears, the residue rapidly convergesnear zero.
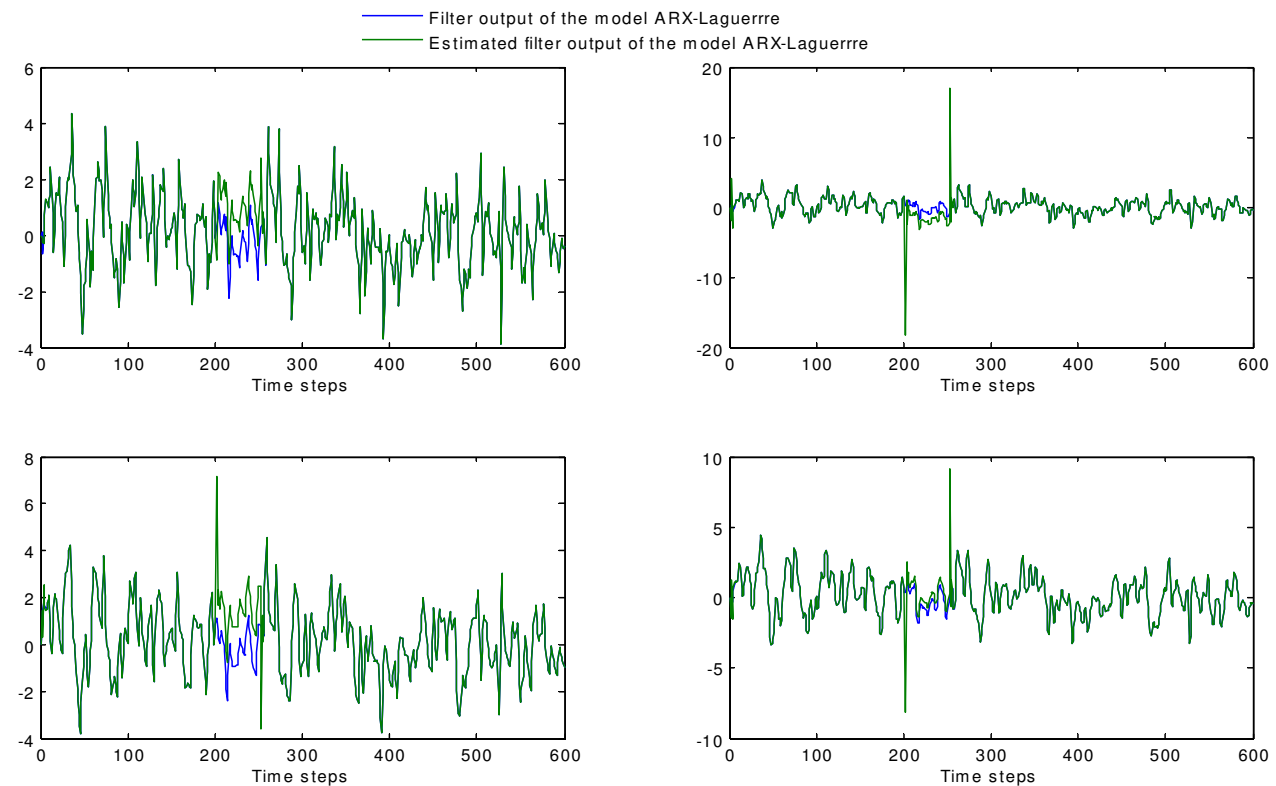

Figure 5. Evolution of $\widehat{X}(k)$ and $X(k)$ 


\section{Conclusion}

The proposed method estimates the outputs vector of Laguerre filters $\hat{X}(k)$ by introducing a proportional gain based on the newARX-Laguerre linear representation. The estimation is based on the input and the output of the system used to build an observer able to generate a default indicator signal called residual signal calculated as the difference between actual values (states or outputs) and their estimated.

The used example demonstrated the ability of the designed observer to estimate the outputs vector of Laguerre filters $\hat{X}(k)$ and to detect the defaults in the system. The use of a single observer for diagnostic can detect defaults but doesn't allow its location. For the isolation of any defaults, other observers structures can be used. The interest of these structures is that each observer can be sensitive to a set of faults and not for all. These structures are used to locate actuator defaults and / or sensor defaults.

\section{REFERENCES}

1. BOUZRARA, K., T. GARNA, H. MESSAOUD, J. RAGOT, Online Identification of the ARX Model Expansion on Laguerre Orthonormal Bases With Filters on Model Input and Output, International Journal of Control, vol. 86, 2013, pp. 369-385.

2. BOUZRARA, K., T. GARNA, H. MESSAOUD, J. RAGOT, Decomposition of an ARX Model on Laguerre Orthonormal Bases, ISA Transactions, vol. 51, 2012, pp. 848-860.

3. VAN SCHRICK, D., PI-Observer-based Reconstruction of Effect-variables and Construction of Characteristic Curves, at the 5-th Asian Control Conference, 2004, pp. 937-942.

4. R. MALTI, Représentation de Systèmes Discrets sur la Base des Filtres Orthogonaux - Application à la Modélisation de Systèmes Dynamiques Multivariables, $\mathrm{PhD}$ thesis, Institut National Polytechnique de Loraine, France, 1999.
5. BEDOUI, H., K. BEN OTHMAN, Multiobserver for Uncertain Output Nonlinear Systems, in 8-th International Conference on Applied Mathematics, Simulation, Modelling (ASM '14), Florence, Italy, 2014, pp. 354-359.

6. BORNE, P., G. DAUPHIN-TANGUY, J. P. RICHARD, G. ROTELLA, I. ZAMBETTAKIS, Commande et Optimisation des Processus, Collection Méthodes et Techniques de l'Ingénieur, Editions Technip, 1990.

7. HE, Y.-Y., X.-J. MA, Z.-Q. SUN, Analysis and Design of Fuzzy Controller and Fuzzy Observer, IEEE Transactions on Fuzzy Systems, vol. 6, 1998, pp. 41-51.

8. IKEDA, T., K. TANAKA, H. O. WANG, Robust Stabilization of a Class of Uncertain Nonlinear Systems via Fuzzy Control: Quadratic Stabilizability, Ho Control Theory, and Linear Matrix Inequalities, IEEE Transactions on Fuzzy Systems, vol. 4, 1996, pp. 1-13.

9. CHEN, J., C. LOPEZ-TORIBIO, R. PATTON, Fuzzy Observers for Nonlinear Dynamic Systems Fault Diagnosis," in Proceedings of the 37-th IEEE Conference on Decision and Control, 1998, pp. 84-89.

10. BEDOUI, H., K. BEN OTHMAN, A. KHEDHER, Fault Detection and Isolation of Fuzzy System with Uncertain Parameters Using the Bounded Approach, WSEAS Transactions on Systems and Control,vol. 9, art. 28, 2014, pp. 269-276.

11. AitOUCHE, A., L. BELKOURA, A. JOUNI, S. C. OLTEANU, Embedded P.E.M. Fuel Cell Stack Nonlinear Observer by Means of a Takagi-Sugeno Approach, Studies in Informatics and Control, ISSN 1220-1766, vol. 24(1), 2015, pp. 61-70.

12. OTSUKA, N., T. SOGA, Stabilizability Conditions for Switched Linear Systems with Constant Input via Switched Observer, Studies in Informatics and Control, ISSN 1220-1766, vol. 22(1) 2013, pp. 7-14. 